\title{
BioMimics 3D vascular stent system for femoropopliteal interventions
}

\section{Two-year results from the real-world multicenter MIMICS-3D registry}

\author{
Michael Lichtenberg ${ }^{1}$ (D), Thomas Zeller², Peter Gaines ${ }^{3}$, and Michael Piorkowski ${ }^{4}$ \\ on behalf of the MIMICS-3D investigators \\ ${ }^{1}$ Vascular Center, Klinikum Hochsauerland, Arnsberg, Germany \\ ${ }^{2}$ Universitätsklinikum Freiburg Herzzentrum, Bad Krozingen, Germany \\ ${ }^{3}$ Sheffield Hallam University, Sheffield, UK \\ ${ }^{4}$ Cardioangiologisches Centrum Bethanien, Frankfurt, Germany
}

\begin{abstract}
Summary: Background: The MIMICS-3D study aimed to assess the safety and effectiveness of the BioMimics 3D Vascular Stent System for the treatment of symptomatic femoropopliteal artery disease in a real-world patient population. Patients and methods: Consecutive participants who were scheduled for implantation of the BioMimics 3D stent were enrolled in the prospective, observational, multicenter study. The primary effectiveness outcome was freedom from clinically driven target lesion revascularization at 12 months and the primary safety outcome was a composite of major adverse events comprising death, major target limb amputation, or clinically driven target lesion revascularization at 30 days. Outcomes through 24 months are reported. Results: A total of 507 patients ( $70 \pm 10$ years, $65.5 \%$ male sex) were enrolled and treated with the study stent. $24.0 \%$ had critical limb-threatening ischemia, lesion length was $127 \pm 92 \mathrm{~mm}$, and $56.8 \%$ of lesions were totally occluded. The Kaplan-Meier (KM) estimate of freedom from clinically driven target lesion revascularization at twelve-months was $90.6 \%(95 \% \mathrm{Cl}: 87.9 \%-93.3 \%)$ and the 30 -day primary safety outcome occurred in $1.2 \%(95 \% \mathrm{Cl}: 0.5 \%-2.7 \%)$ of participants. At 24 months, clinical improvement was achieved in $86.6 \%$ and the KM estimate of freedom from clinically driven target lesion revascularization was $82.8 \%$ (95\% Cl: $79.4 \%-86.4 \%)$. The KM estimate of freedom from loss of primary patency according to PSVR $>2.4$ was $78.6 \%$ (95\% Cl: $74.7 \%-82.4 \%)$. Survival distribution functions regarding primary patency were lower with long lesions $(>150 \mathrm{~mm}$; log-rank $\mathrm{p}<0.001)$ but did not differ significantly between participants with or without critical limb-threatening ischemia (log-rank $\mathrm{p}=0.07$ ). Conclusions: Endovascular treatment of atherosclerotic femoropopliteal lesions with the BioMimics 3D Vascular Stent System is efficacious and safe in a real-world setting.
\end{abstract}

Keywords: endovascular therapy, femoropopliteal, patency, peripheral artery disease, swirling flow, self-expanding stent, helical stent, superficial femoral artery, target lesion revascularization

\section{Introduction}

Endovascular techniques have developed to treat patients with peripheral arterial disease (PAD), and there has been considerable innovation focus on improving both treatment success and durability. Stent-based therapy has been shown to be superior to standard balloon angioplasty [1] when treating disease affecting the superficial femoral and popliteal arteries, and stents are used in nearly 50\% of interventions [2].

Although there is a trend for non-stent interventions to "leave nothing behind", stents are quite helpful particularly concerning complex lesions including occlusions, calcification, or dissections. Femoropopliteal artery disease is common in patients with symptomatic PAD and those affected by chronic limb threatening ischaemia (CLTI) lesions are typically elderly with long, highly calcified disease. These lesion characteristics along with adverse flow conditions result in high rates of restenosis and the need for reintervention to maintain patency. That reintervention adds further risk for the patient and is a financial burden for health care systems. Modern generation non-drug eluting and drug eluting nitinol stents for the treatment of femoropopliteal artery lesions have shown encouraging patency rates and long-term freedom from restenosis rates in different prospective trials $[3,4,5,6,7,8,9,10,11,12,15,16]$.

The helical geometry of the BioMimics 3D stent (Veryan Medical Ltd., Horsham, UK) is designed to render the 
curvature of the vessel helical to impart swirling flow and to elevate wall shear stress which has been shown to prevent intimal hyperplasia and restenosis [3]. Therefore, we aimed to assess effectiveness and safety of the BioMimics 3D stent in a real-world setting.

\section{Patients and methods}

\section{Study design}

The aim of the MIMICS-3D Registry is to assess the performance of the BioMimics 3D Vascular Stent System in a real world setting through 36 months. Outcomes through 24 months are reported in this article. It was designed as a prospective, observational, multicenter study. From September 2016 to June 2018, eligible patients with symptomatic femoropopliteal artery disease who were scheduled for treatment with BioMimics 3D were consecutively enrolled. Patients' written informed consent regarding study participation was obtained prior to the index procedure. Follow-up examinations were planned for 30 days, 12, 24, and 36 months from index procedure. Adverse events were adjudicated by an independent Clinical Events Committee. The study was approved by the responsible ethics committee and the local ethics committees of all participating sites. It complies with the Declaration of Helsinki and is registered with ClinicalTrials.gov (Identifier: NCT02900924).

\section{Outcomes and definitions}

The primary effectiveness endpoint was freedom from clinically driven target lesion revascularization (CDTLR) at 12 months. Revascularization was considered clinically driven in patients with objective evidence of recurrent symptoms associated with an angiographic determination of $\geq 50 \%$ stenosis and new distal ischemic signs i.e: worsening ankle brachial index (ABI) or worsening Rutherford Category associated with the index limb. The primary safety endpoint was a composite of death, major target limb amputation, or CDTLR through 30 days. Major amputation was defined as above transmetatarsal amputation.

Secondary outcome measures were acute technical success, defined as residual diameter stenosis $\leq 30 \%$ at the end of the procedure and acute procedural success, defined as 72-hour incidence of both acute technical success and absence of severe adverse events including death, stroke, myocardial infarction, acute limb ischemia, treated segment thrombosis, or need for bypass graft or emergency index limb vascular surgery. Other secondary endpoints were death, major target limb amputation, and CDTLR at 30 days, 12, 24, and 36 months, cumulative incidence of primary patency at 12,24 , and 36 months, assessed by duplex ultrasonography (peak systolic velocity ratio [PSVR] $\leq 2.4$ ), clinical improvement (reflected by change in Rutherford category), hemodynamic improvement expressed as change in ABI from baseline, and incidence of stent fractures. Stent fractures were defined as clear interruption of stent struts observed by biplanar radiography and were assessed by the implanting physician.

\section{Study population}

A real-world clinical population of patients $\geq 18$ and $\leq 85$ years of age with symptomatic peripheral artery disease due to atherosclerotic femoropopliteal lesions scheduled for treatment with BioMimics 3D were eligible for inclusion in the study. Major exclusion criteria were failure to cross the lesion with a guidewire and/or balloon catheter and insufficient dilation to allow passage of the study device, intolerance to antiplatelet and/or anticoagulation therapy, hypersensitivity to nickel-titanium, bleeding diathesis, severe hypertension, renal failure, and pregnancy or breastfeeding.

\section{Study stent and stent implantation}

The BioMimics 3D Vascular Stent System (BioMimics 3D) (Veryan Medical Ltd., Horsham, UK) consists of a selfexpanding, laser-cut nitinol stent with 3D helical centerline geometry and an over-the-wire stent delivery system (Figures 1 and 2). Previous studies intended to gain regulatory approval in Europe, the US and Japan have shown good safety and efficacy of BioMimics 3D albeit in a well-defined cohort of patients. Details about the stent system have been described elsewhere $[3,4,5]$. The helical centerline curvature of the stent is stored within the shape memory of the nitinol alloy during manufacture. The stent is designed to impart a helical shape to vessel morphology which both accommodates prevalent biomechanical stresses particularly during hip and knee flexion and induces laminar swirling flow which increases wall shear stress (WSS) on the vessel wall.

The BioMimics 3D Vascular Stent System is CE marked and has FDA approval and was intended to be used according to the manufacturer's instructions for use. The stent is indicated for the treatment of the SFA and/or the proximal popliteal artery. Vascular access, lesion preparation, poststenting treatment, and concomitant antiplatelet and anticoagulant medication were conducted according to centers' standard of care.

\section{Statistical analysis}

We report continuous variables as mean \pm SD and categorical variables as absolute and relative frequencies. Primary endpoints were analyzed using both the modified intention-to-treat (ITT) and the per protocol (PP) study population. All other analyses referred to the ITT population. Primary endpoints are presented as frequency and percentage. The corresponding 95\% confidence interval (CI) was calculated using the Agresti-Coull method. Kaplan-Meier (KM) analysis was performed to assess cumulative incidence of freedom from CDTLR, primary patency, 


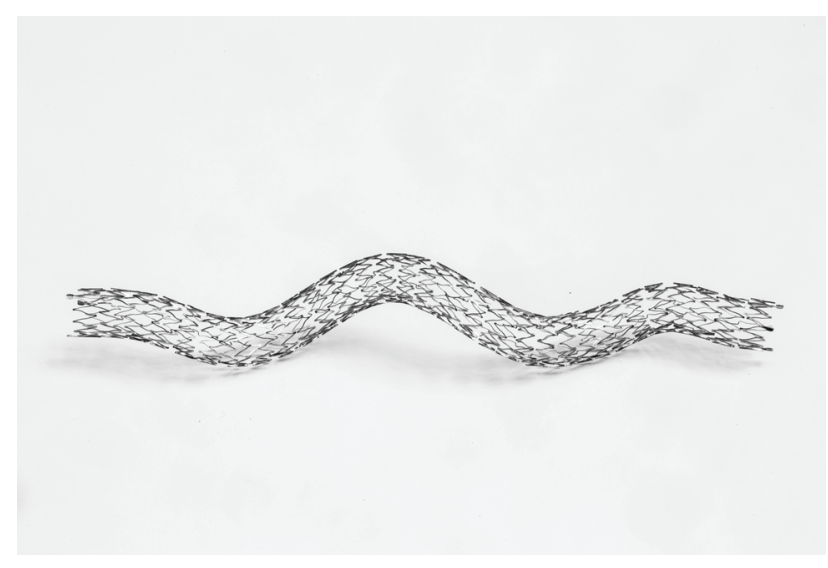

Figure 1. BioMimics 3D.

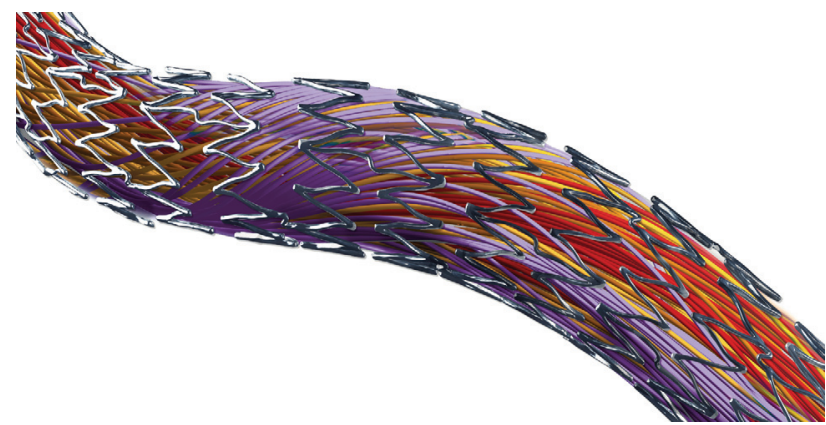

Figure 2. BioMmics 3D with computation fluid dynamics representation of swirling flow.

amputation free survival, and freedom from death throughout 24 months. Results are presented as parameter estimates and their corresponding 95\% CI survival curves were compared using log-rank test. A 2-sided value of $\mathrm{p}<0.05$ was considered to indicate statistical significance. We analyzed data by using RStudio Version 1.2.5042 and SAS Version 9.4.

\section{Results}

\section{Study population and treatment}

A total of 507 consecutive patients with 518 femoropopliteal artery lesions were enrolled over 21 months at 23 sites in Germany, Belgium, the Netherlands, and Sweden. All participants underwent endovascular angioplasty and stent implantation using the BioMimics 3D Vascular Stent System. Twenty-four percent of participants had chronic limb-threatening ischemia (CLTI), 37\% were diabetics and $38 \%$ current smokers (Table I). Almost all lesions were femoropopliteal (91\%), one third (38\%) were moderate or severely calcified (Grade 2 and 4 according to a peripheral arterial calcium scoring system [PACSS] [6]), 57\% were totally occluded and $10 \%$ of disease was due to restenosis after balloon angioplasty. The mean lesion length was $127 \pm 92 \mathrm{~mm}$ (Table II). Pre-dilation was conducted in $88 \%$ of lesions. Concomitant drug-coated balloon angioplasty was performed in $24 \%$ for lesion preparation and in $26 \%$
Table I. Baseline demographics and clinical characteristics

\begin{tabular}{|c|c|}
\hline Age, y & $70.1 \pm 10$ \\
\hline Men & $332 / 507(65.5)$ \\
\hline Diabetes mellitus & $187 / 507(36.9)$ \\
\hline Insulin & $82 / 507(16.2)$ \\
\hline Hypercholesterolemia/dyslipidemia & $324 / 507$ (63.9) \\
\hline Hypertension & 434/507 (85.6) \\
\hline Smoking (current and former) & $345 / 507(68.0)$ \\
\hline Current & $191 / 507(37.7)$ \\
\hline Former & $154 / 507(30.4)$ \\
\hline Previous myocardial infarction & $56 / 507(11.0)$ \\
\hline $\begin{array}{l}\text { Previous cerebrovascular accident } \\
\text { including stroke or TIA }\end{array}$ & $57 / 507(11.2)$ \\
\hline Previous CABG & $58 / 507(11.4)$ \\
\hline Renal disease & 42/507 (8.3) \\
\hline Dialysis & $10 / 507(2)$ \\
\hline \multicolumn{2}{|l|}{ Rutherford category (missing data $=3$ ) } \\
\hline 0 & $2 / 504(0.4)$ \\
\hline 1 & $6 / 504(1.2)$ \\
\hline 2 & $86 / 504(17.1)$ \\
\hline 3 & $289 / 504(57.3)$ \\
\hline 4 & $38 / 504(7.5)$ \\
\hline 5 & $72 / 504(14.3)$ \\
\hline 6 & $11 / 504(2.2)$ \\
\hline
\end{tabular}

for post-stenting treatment (Table III). A total of 398 participants (79\%) completed the 24-month follow-up.

\section{Primary endpoints}

Freedom from CDTLR at 12 months was achieved in $89.4 \%$ (95\% CI: $86.2 \%-92.0 \%$ ) of the participants (Table IV). At 30 days after index procedure, major adverse events occurred in 6 of 495 participants (1.2\%). Two of them underwent CDTLR, two needed major target limb amputation and two died due to myocardial infarction.

\section{Secondary endpoints}

Acute technical success was achieved in $99.0 \%$ of lesions, and acute procedural success in $97.4 \%$ of participants. The KM estimate of freedom from CDTLR was $82.8 \%$ (95\% CI: $79.4 \%-86.4 \%$ ) at 24 months. Through 24 months, CDTLR for patients with either CLTI or claudication did not show any statistically significant difference (log-rank $\mathrm{p}=0.1$ ) (Figure 3), although there is slight separation of the curves through that period in favour of claudication.

The KM estimate of primary patency according to PSVR $>2.4$ was $87.7 \%$ (95\% CI: $84.6 \%-90.7 \%$ ) and $78.6 \%$ (95\% CI: $74.7 \%-82.4 \%$ ) at 12 and 24 months, respectively (Figure 4). Primary patency was lower in long lesions compared to lesions $\leq 150 \mathrm{~mm}$ (log-rank $\mathrm{p}<0.001)$. Although there was no statistically significant difference between patients suffering from CLTI rather than claudication (log rank $\mathrm{p}=0.07$ ) again there is slight separation of the curves 
Table II. Baseline lesion characteristics

\begin{tabular}{lc}
\hline Number of lesions & $\mathrm{N}=518$ \\
\hline Proximal lesion boundary & \\
Iliac artery & $5 / 518(1.0)$ \\
Common femoral artery & $2 / 518(0.4)$ \\
Ostial SFA & $43 / 518(8.3)$ \\
Proximal SFA & $95 / 518(18.3)$ \\
Mid SFA & $169 / 518(32.6)$ \\
Distal SFA & $159 / 518(30.7)$ \\
Popliteal artery & $44 / 518(8.5)$ \\
Lesion length, mm & $127 \pm 92$ \\
Calcification ${ }^{2}$ (missing data=2) & \\
Grade 0 & $91 / 516(17.6)$ \\
Grade 1 & $152 / 516(29.5)$ \\
Grade 2 & $125 / 516(2.4)$ \\
Grade 3 & $76 / 516(14.7)$ \\
Grade 4 & $72 / 516(14.0)$ \\
Diameter stenosis, \% & $94.6 \pm 8.0$ \\
Total occlusion & $294 / 518(56.8 \%)$ \\
Target lesion type & \\
De novo & \\
Restenotic ${ }^{b}$ & $467 / 518(90.2 \%)$ \\
Number of patent runoff vessels & $51 / 518(9.8 \%)$ \\
0 & \\
1 & $22 / 507(4.3)$ \\
3 & $107 / 507(21.1)$ \\
\hline Notes. Contnous & $169 / 507(33.3)$ \\
\hline & $209 / 507(41.2)$ \\
\hline
\end{tabular}

Notes. Continuous variables are presented as means \pm standard deviation and categorical variables as counts/sample (percentage). ${ }^{a}$ According to peripheral arterial calcium scoring system (PACSS). ${ }^{\text {b }}$ Restenosis after preceding balloon angioplasty. SFA: superficial femoral artery.

Table III. Procedure characteristics

\begin{tabular}{lc}
\hline Lesion preparation & \\
Pre-dilation (standard PTA balloon) & $454 / 518(87.6)$ \\
DCB & $122 / 518(23.6)$ \\
Cutting balloon & $16 / 518(3.1)$ \\
BMS & $7 / 518(1.4)$ \\
Thrombectomy & $37 / 518(7.1)$ \\
Atherectomy & $39 / 518(7.5)$ \\
Number of stents (BioMimics 3D) deployed & \\
1 & $396 / 518(76.4)$ \\
2 & $95 / 518(18.3)$ \\
3 & $20 / 518(3.9)$ \\
4 & $7 / 518(1.4)$ \\
Stented length, mm & $130.8 \pm 79.2$ \\
Post treatment after stent placement & \\
Post-dilation (standard PTA balloon) & $353 / 518(68.1)$ \\
DCB & $137 / 518(26.4)$ \\
BMS & $9 / 518(1.7)$ \\
DES & $2 / 518(0.4)$ \\
Covered stent & $5 / 518(1.0)$ \\
Thrombectomy & $3 / 518(0.6)$ \\
\hline
\end{tabular}

Notes. Continuous data are presented as means \pm standard deviation and categorical variables as counts/sample (percentage). BMS: bare metal stent; DCB: drug-coated balloon; DES: drug-eluting stent. in favour of claudication out to 24 months. There was no difference in patency when a paclitaxel DCB was used in addition to the BioMimics 3D stent (Figure 5).

At 24 months, clinical improvement by at least one Rutherford category was observed in $86.6 \%$ of the participants and ankle-brachial index improved from $0.6 \pm 0.3$ at baseline to $0.9 \pm 0.2(\mathrm{n}=304)$. KM estimates of freedom from major target limb amputation was $98.5 \%$ (95\% CI: $97.3 \%-99.6 \%)$ and freedom from death was $89.4 \%(95 \%$ CI: $86.6 \%-92.2 \%)$.

Stent fracture analysis was performed in case of target lesion revascularization. Out of 674 stents implanted there were 3 confirmed stent fractures classified as multiple, single strut fractures without complete transection or displacement of the stent. For one of the confirmed stent fractures, the strut factures were co-located with a focal aneurysm. However, none of them was identified to be related to a deficiency in the device. Serious adverse events through 24 months are displayed in (Electronic Supplementary Material [ESM] 1).

\section{Discussion}

The results of this MIMICS-3D Registry show a KM estimate of freedom from loss of primary patency at 12 and 24 months of $87.7 \%$ and $78.6 \%$ respectively.

The BioMimics 3D Vascular Stent System, designed to treat symptomatic PAD affecting the femoropopliteal segment, has a unique helical centerline that is intended to impart a helical shape to the artery for inducing swirling flow and so increasing wall shear stress. High wall shear has been shown to be associated with a low prevalence of atherosclerosis and a reduced risk of restenosis [17, 18, 19, 20]. The biological mechanisms are complex but swirling flow, and the resultant elevated wall shear stress affects all the pathways (thrombosis, inflammation, reendothelialization, altered smooth muscle cells) that result in neointimal hyperplasia [21]. The device has previously been shown in the MIMICS-RCT randomised trial to perform better than a straight stent. The MIMICS-2 IDE study recruited 271 subjects from 43 investigational sites across 3 continents and confirmed good outcomes at 12 and 24 months with a Kaplan-Meier freedom from loss of primary patency of $83.1 \%$ and $69.6 \%$, and freedom from clinically driven target lesion revascularization of $88.7 \%$ and $84.2 \%$ respectively. These 2 studies were performed in cohorts of predominantly claudicants with mean lesion lengths of 65.8 and $81.2 \mathrm{~mm}$. This prospective, multicenter observational registry evaluated effectiveness and safety of the BioMimics 3D stent system in a real-world setting, designed to include patients with CLTI and longer, more challenging lesions.

The MIMICS-3D registry recruited 507 patients from 23 sites and the treatment of these patients was representative of typical interventional practices: the mean age was $70.1 \mathrm{yrs} ; 24 \%$ of patients had CLTI, $36.9 \%$ of whom 
Table IV. Primary endpoints

\begin{tabular}{lcr}
\hline Effectiveness & ITT & PP \\
\hline Freedom from CDTLR at 12 months & $89.4 \%(396 / 443)$ & $90.6 \%(367 / 405)$ \\
Safety & $95 \% \mathrm{Cl}: 86.2 \%-92.0 \%$ & $95 \% \mathrm{Cl}: 87.4 \%-93.1 \%)$ \\
Composite of MAE ${ }^{\mathrm{a}}$ at 30 days & $1.2 \%(6 / 495)$ & $1.1 \%(5 / 452)$ \\
& $95 \% \mathrm{Cl}: 0.5 \%-2.7 \%$ & $95 \% \mathrm{Cl}: 0.4 \%-2.6 \%$ \\
Death & $0.4 \%(2 / 494)$ & $0.4 \%(2 / 451)$ \\
& $95 \% \mathrm{Cl}: 0.01 \%-1.6 \%$ & $95 \% \mathrm{Cl}: 0.01 \%-1.7 \%$ \\
Major amputation (index limb) & $0.4 \%(2 / 494)$ & $0.4 \%(2 / 451)$ \\
& $95 \% \mathrm{Cl}: 0.01 \%-1.6 \%$ & $95 \% \mathrm{Cl}: 0.01 \%-1.7 \%$ \\
CDTLR & $0.4 \%(2 / 493)$ & $0.2(1 / 450)$ \\
& $95 \% \mathrm{Cl}: 0.01 \%-1.6 \%$ & $95 \% \mathrm{Cl}:-0.09 \%-1.4 \%$ \\
\hline
\end{tabular}

Notes. ${ }^{a}$ Composite of all-cause death, major target limb amputation, and clinically driven target lesion revascularization. CDTLR: clinically driven target lesion revascularization; ITT: intention-to-treat study population; MAE: major adverse event; PP: per protocol study population.

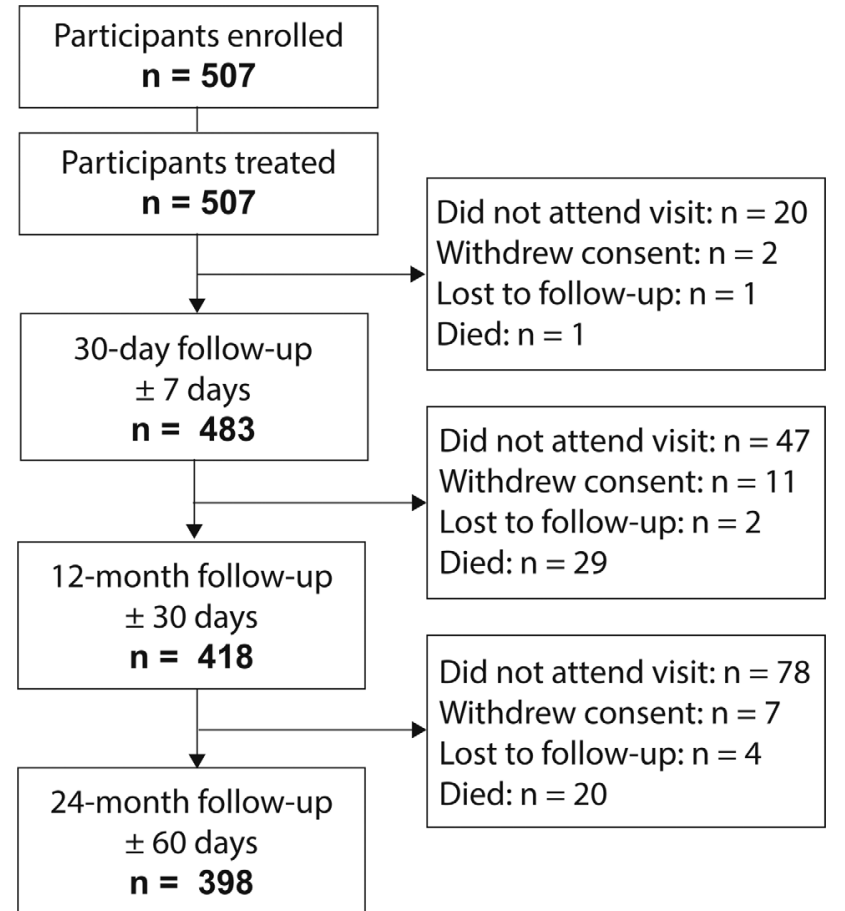

Figure 3. Participant flow.

suffered from diabetes; the mean lesion length was $127 \pm 92 \mathrm{~mm}, 56.8 \%$ of which were total occlusions. Despite the considerable comorbidity and technical challenges, the high acute technical and procedural success rates, and 30-day freedom from MAE (composite of death, major target limb amputation, or CDTLR) of $98.8 \%$, reinforce the procedural benefits of the device.

There is a now an extensive published literature on the use of stents in the femoropopliteal segment, a review of which lies outside the remit of this article. However, since 2010 there have been three meta-analyses from which a comparison can be drawn between the performance of the helical BioMimics 3D stent and that of other bare metal nitinol stents with straight centerlines. The authors are not aware of any other high-quality studies subsequent to these publications that might substantially change the basis for comparison. Acin et al. investigated the outcome of bare metal nitinol stents by reviewing 4 high quality randomized
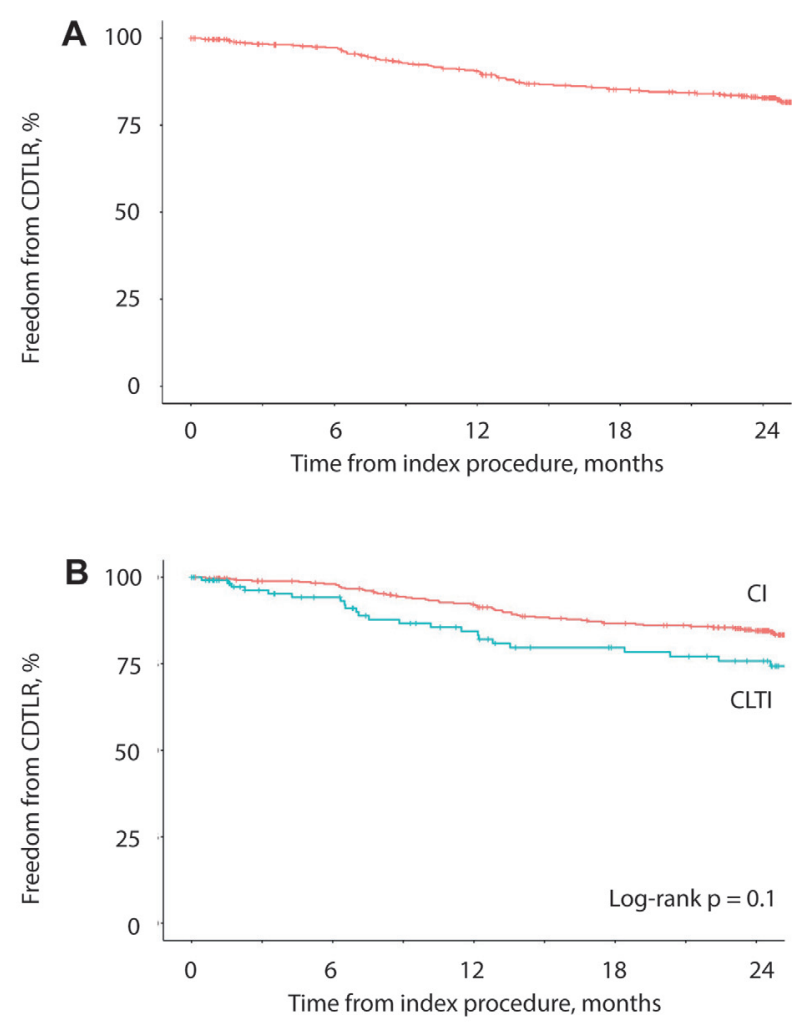

At risk

$\begin{array}{rrrrr}\text { All } & 507 & 445 & 400 & 313 \\ \text { Cl } & 383 & 352 & 324 & 256 \\ \text { CLTI } & 121 & 90 & 73 & 55\end{array}$

Freedom from CDTLR

$\begin{array}{rlll}\text { All } & 97.2 \% & 90.6 \% & 82.8 \% \\ \text { Cl } & 98.1 \% & 92.2 \% & 84.6 \% \\ \text { CLTI } & 94.2 \% & 84.4 \% & 75.8 \%\end{array}$

Figure 4. Kaplan-Meier survival curves for freedom from clinically driven target lesion revascularization through 24 months after BioMimics 3D stent placement shown for the total study cohort (A) and for the comparison between participants with intermittent claudication or chronic limb-threatening ischemia (B). Cl: intermittent claudication; CLTI: chronic limb-threatening ischemia.

trials that fulfilled their criteria. From their data the 12-month patency was $73 \%$ and TLR rate was $17 \%$ [22]. Rocha-Singh et al. undertook a patient level meta-analysis 

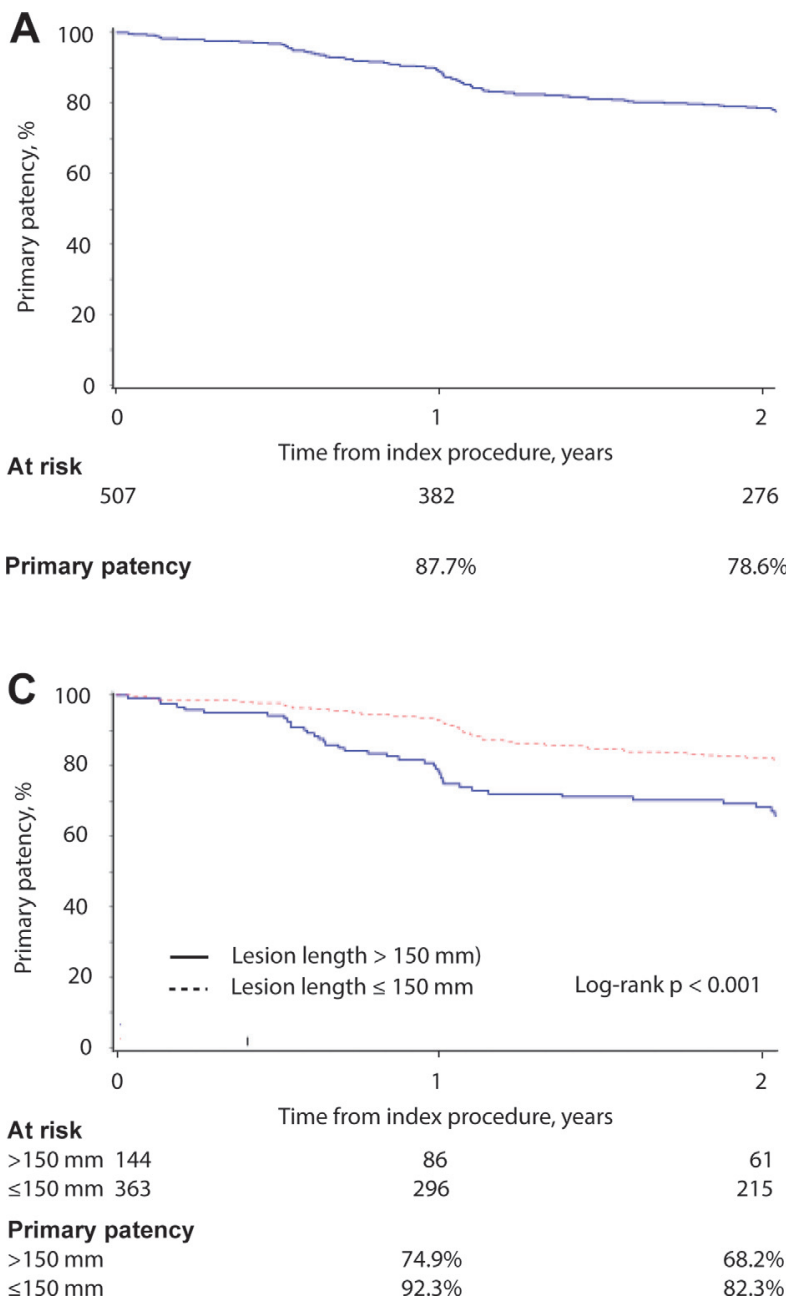
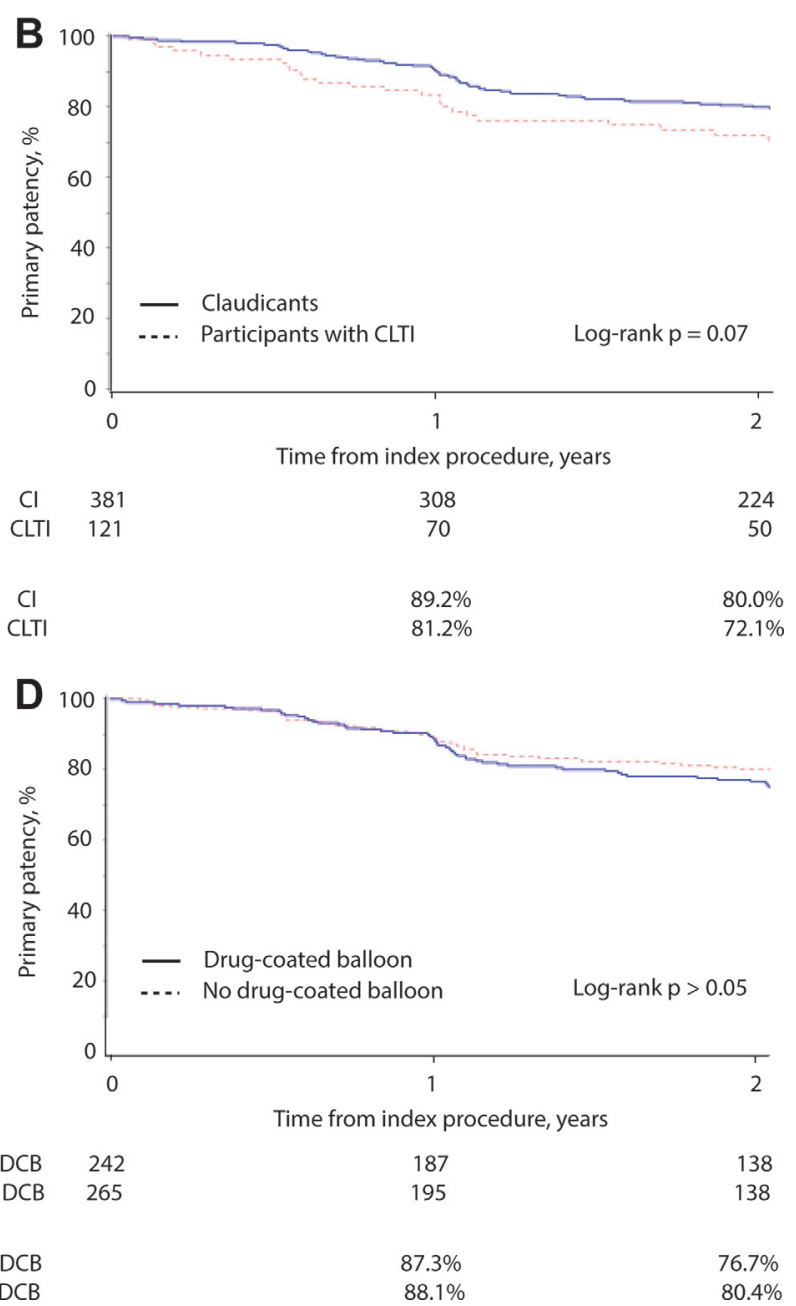

Figure 5. Kaplan-Meier survival curves for primary patency through two years after BioMimics 3D stent placement (A) showing comparisons of intermittent claudication versus chronic limb-threatening ischemia (B), lesion length of >150 mm versus $\leq 150 \mathrm{~mm}(\mathrm{C})$, and concomitant use versus non-use of drug-coated balloons (D). DCB: drug-coated balloon; Cl: intermittent claudication; CLTI: chronic limb-threatening ischemia.

of 999 subjects undergoing primary nitinol stent implantation from 6 large prospective investigations using devices approved by the FDA [23]. The mean age was 67.7 years and subjects were claudicants (mean Rutherford Clinical category 2.7, range: 2.6-2.8). A mean lesion length of $83.8 \mathrm{~mm}$ was treated resulting in a 12-month primary patency of $69.8 \%$ and TLR rate of $9.2-19.7 \%$. The subject's initial ABI and lesion length predicted both patency and TLR. These outcomes are comparable to the data from the MIMICS-2 IDE study and are impressive when compared to those for straight nitinol stents, particularly since the subjects were elderly, nearly a quarter had CLTI and the lesions were long. The 12- and 24-month KM freedom from CDTLR rates of $90.6 \%$ and $82.8 \%$ are similarly impressive when compared to results for straight nitinol stents and suggest that there is a clinical benefit to the use of a stent with a helical centerline to generate swirling flow. It is generally appreciated that revascularisation is less durable and requires more re-intervention in patients suffering from CLTI rather than claudication. The data from this study show minimal difference in outcome between the two groups.

Half the subjects in this study had adjunctive treatment with a paclitaxel drug coated balloon (DCB) in addition to the stent. There was no advantage in terms of patency or CDTLR conferred by the DCB. The reason for this is unclear at this stage and cannot be answered by this trial. However, given that swirling flow and increased wall shear stress affect all aspects of the biological response that results in neointimal hyperplasia, including an effect upon the vascular smooth muscle cells, one could assume that paclitaxel offers no incremental contribution to the process [21]. Such a finding that a DCB adds no further benefit would have potentially important cost considerations.

Contemporary stents intended for use within the femoropopliteal segment have reported fractures rates of 0\% [7], $0.4 \%$ [8], 0.5\% [9], 2.0\% [10], 3.1\% [11]. The BioMimics 3D stent's ability to shorten with the vessel during hip and knee flexion is intended to prevent kinking and fatigue fractures that can affect straight stents unable to shorten within the stented vessel. In this study, only 3 fractures were identified, however none of them was identified to be related to a deficiency in the device, and only lesions which needed revascularization were examined. No stent fractures had been observed through 24 months in the earlier MIMICS-RCT [4] and MIMICS-2 studies [5]. In previous studies of other femoropopliteal stents, fracture rate increased with stented length and was associated with 
297 of 343 participants $(86.6 \%)$ improved by

$\geq 1$ Rutherford category

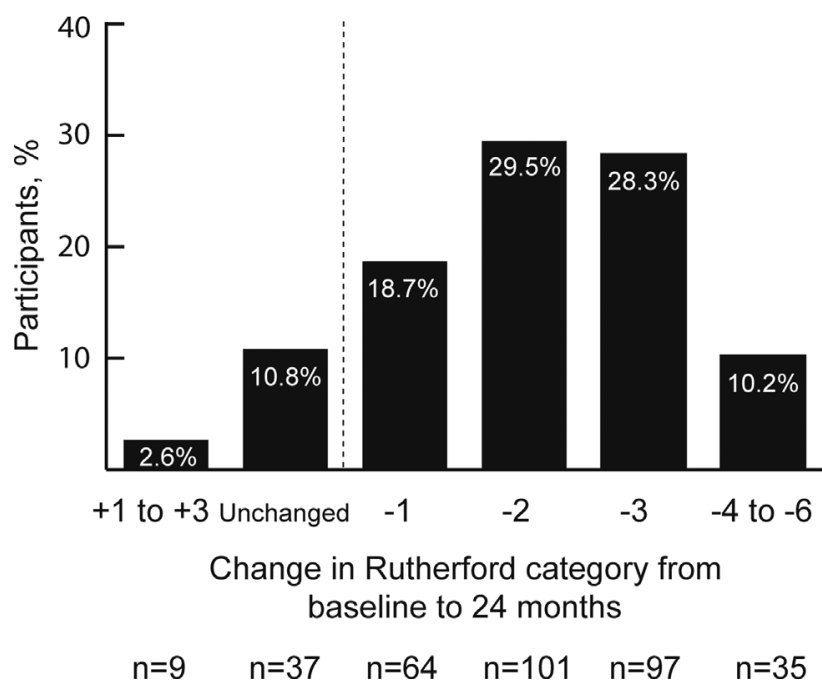

Figure 6. Clinical improvement at 24 months.

involvement of the distal superficial femoral artery [12]. Overall, patients may benefit from novel stent designs with increased flexibility along with sufficient radial force. Scheinert et al. found significantly lower Kaplan-Meier estimates of 12-month primary patency in patients with stent fractures $[12,13,14]$. This was not confirmed by Davaine et al. [13] and Iida et al. reported that the effect of stent fractures on patency only persists over the first two years [14]. Whilst the overall effect of differing grades of fracture remains unclear, it is the authors' belief that high grade fractures are likely to confer adverse outcomes upon the patient and stent selection should be undertaken to mitigate this risk.

\section{Limitations}

Comparison of results with other studies should be conducted cautiously because of differences in participant, lesion, and procedure characteristics. In this study there were no restrictions on concomitant pre- and post-stenting treatment and the implications of such variables cannot be clearly delineated. Validity of 24-month results is limited due to considerable loss of follow-up. Follow up was conducted according to centers' standard without adjudication by core laboratory but all primary endpoint-determining events were subjected to clinical event committee adjudication.

\section{Conclusions}

The MIMICS-3D study confirms that a clinically-representative, real world patient population suffering from symptomatic disease of the femoropopliteal segment can be safely and effectively treated with the BioMimics 3D stent system.

\section{Electronic supplementary material}

The electronic supplementary material (ESM) is available with the online version of the article at https://doi.org/ 10.1024/0301-1526/a000980

ESM 1. Device or procedure related serious adverse events at 24 months (Table)

\section{References}

1. Chowdhury MM, McLain AD, Twine CP. Angioplasty versus bare metal stenting for superficial femoral artery lesions. Cochrane Database Syst Rev. 2014;2014(6):CD006767.

2. Banerjee S, Jeon-Slaughter $H$, Armstrong EJ, Bajzer C, Abu-Fadel M, Khalili $\mathrm{H}$, et al. Clinical outcomes and cost comparisons of stent and non-stent interventions in infrainguinal peripheral artery disease: Insights from the Excellence in Peripheral Artery Disease (XLPAD) registry. J Invasive Cardiol. 2019;31(1):1-9.

3. Sullivan TM, Zeller T, Nakamura M, Caro CG, Lichtenberg M. Swirling flow and wall shear: evaluating the BioMimics 3D helical centerline stent for the femoropopliteal segment. Int J Vasc Med. 2018;2018:9795174.

4. Zeller T, Gaines PA, Ansel GM, Caro CG. Helical centerline stent improves patency: two-year results from the randomized mimics trial. Circ Cardiovasc Interv. 2016;9(6).

5. Sullivan TM, Zeller T, Nakamura M, Gaines PA. Treatment of femoropopliteal lesions with the BioMimics 3D vascular stent system: two-year results from the MIMICS-2 trial. J Endovasc Ther. 2020;1526602820980419.

6. Rocha-Singh KJ, Zeller T, Jaff MR. Peripheral arterial calcification: prevalence, mechanism, detection, and clinical implications. Catheter Cardiovasc Interv. 2014;83(6):E212-20.

7. Garcia L, Jaff MR, Metzger C, Sedillo G, Pershad A, Zidar F, et al. Wire-interwoven nitinol stent outcome in the superficial femoral and proximal popliteal arteries: twelvemonth results of the SUPERB trial. Circ Cardiovasc Interv. 2015:8(5).

8. Matsumura JS, Yamanouchi D, Goldstein JA, Pollock CW, Bosiers M, Schultz GA, et al. The United States StuDy for EvalUating EndovasculaR TreAtments of Lesions in the Superficial Femoral Artery and Proximal Popliteal By uslng the Protégé EverfLex NitInol STent SYstem II (DURABILITY II). J Vasc Surg. 2013;58(1):73-83.e1.

9. Ohki T, Angle JF, Yokoi H, Jaff MR, Popma J, Piegari G, et al. One-year outcomes of the U.S. and Japanese regulatory trial of the Misago stent for treatment of superficial femoral artery disease (OSPREY study). J Vasc Surg. 2016;63(2): 370-6.e1.

10. Gray WA, Feiring A, Cioppi M, Hibbard R, Gray B, Khatib Y, et al. S.M.A.R.T. self-expanding nitinol stent for the treatment of atherosclerotic lesions in the superficial femoral artery (STROLL): 1-year outcomes. J Vasc Interv Radiol. 2015;26(1): 21-8.

11. Laird JR, Jain A, Zeller T, Feldman R, Scheinert D, Popma JJ, et al. Nitinol stent implantation in the superficial femoral artery and proximal popliteal artery: twelve-month results from the complete SE multicenter trial. J Endovasc Ther. 2014;21(2):202-12.

12. Scheinert D, Scheinert S, Sax J, Piorkowski C, Bräunlich S, Ulrich $\mathrm{M}$, et al. Prevalence and clinical impact of stent fractures after femoropopliteal stenting. J Am Coll Cardiol. 2005;45(2):312-5

13. Davaine JM, Quérat J, Guyomarch B, Brennan M, Costargent A, Chaillou $P$, et al. Incidence and the clinical impact of stent fractures after primary stenting for TASC C and D femoropopliteal lesions at 1 year. Eur J Vasc Endovasc Surg. 2013;46(2): $201-12$. 
14. Iida O, Nanto S, Uematsu M, Ikeoka K, Okamoto S, Nagata S. Influence of stent fracture on the long-term patency in the femoro-popliteal artery: experience of 4 years. JACC Cardiovasc Interv. 2009;2(7):665-71.

15. Lichtenberg M, Breuckmann F, Kramer V, Betge S, Sixt S, Hailer B, et al. Effectiveness of the pulsar-18 self-expanding stent with optional drug-coated balloon angioplasty in the treatment of femoropopliteal lesions - the bioflex peace allcomers registry. VASA. 2019;48:425-32.

16. Frank U, Nikol S, Belch J, Boc V, Brodmann M, Carpentier PH, et al. ESVM Guideline on peripheral arterial disease. VASA. 2019;48(Suppl 102):1-79.

17. Caro CG. Discovery of the role of wall shear in atherosclerosis. Arterioscler Thromb Vasc Biol. 2009;29:158-61.

18. Caro CG, Seneviratne A, Heraty KB, Monaco C, Burke MG, Krams R, et al. Intimal hyperplasia following implantation of helical-centreline and straight-centreline stents in common carotid arteries in healthy pigs: Influence of intraluminal flow. J R Soc Interface. 2013;10:20130578.

19. Malek AM, Alper SL, Izumo S. Hemodynamic shear stress and its role in atherosclerosis. JAMA. 1999;282(21):2035-42.

20. Sanmartín M, Goicolea J, García C, García J, Crespo A, Rodriguez J, et al. Influence of shear stress on in-stent restenosis. In vivo study using 3D reconstruction and computational fluid dynamics. Rev Esp Cardiol. 2006;59(1):20-7.

21. Murphy EA, Boyle FJ. Reducing in-stent restenosis through novel stent flow field augmentation. Cardiovasc Eng Technol. 2012;3:353-73.

22. Acin F, de Haro J, Bleda S, Varela C, Esparza L. Primary nitinol stenting in femoropopliteal occlusive disease: a metaanalysis of randomized controlled trials. J Endovasc Ther. 2012;19:585-95.

23. Rocha-Singh KJ, Beckman JA, Ansel G, Lyden SP, Schneider P, Mehta M, et al. Patient-level meta-analysis of 999 claudicants undergoing primary femoropopliteal nitinol stent implantation. Catheter Cardiovasc Interv. 2017;89(7):1250-6.

\section{History}

Submitted: 04.07.2021

Accepted after revision: 06.11.2021

Published online: 08.12.2021

\section{Acknowledgement}

MIMICS-3D Site Principal Investigators

Germany: Volker Sesselman, SRH Zentralklinikum Suhl; Dierk Scheinert, University Hospital Leipzig; Andrej Schmidt,
University Hospital Leipzig; Sebastian Schellong, Städtisches Klinikum Dresden-Friedrichstadt; Sebastian Debus, University Heart Center Hamburg-Eppendorf; Jens Stegemann, KEH Berlin; Ulrich Sunderdiek, Marienhospital Osnabrück; KaiUwe Wagenhofer, Asklepios Klinik Harburg; Robert Klamroth, Vivantes Klinikum Friedrichshain; Alexandra Müller-Öffner, Gefäßpraxis im Tal; Christos Rammos, Univer-

sitätsklinikum Essen; Klaus Hertting, Krankenhaus Buchholz; Gerd Groezinger, Universitätsklinikum Tübingen; Gunnar Tepe, RoMed Klinikum Rosenheim.

Belgium: Peter Goverde, ZNA Stuivenberg Hospital; Willem Willaert, AZ Maria Middelares; Lieven Maene, OLV Hospital; Koen DeLoose, AZ Sint Blasius; Koen Keirse, RZ Helig Hart Tienen; The Netherlands: Michel Reijnen, Rijnstate Hospital.

Sweden: Nuno Dias, Skane University Hospital Vascular Center.

\section{Conflict of interest}

The author(s) declared the following potential conflicts of interest with respect to the research, authorship, and/or publication of this article:

Michael Lichtenberg has received speaker honoraria from Veryan Medical Ltd. and also serves as a consultant for Veryan Medical Ltd. He is the principal investigator of the MIMICS-3D Registry. Thomas Zeller has received speaker honoraria from Veryan Medical Ltd. and also serves as a consultant for Veryan Medical Ltd.

Peter Gaines serves as a consultant for Veryan Medical Ltd.

Michael Piorkowski has received speaker honoraria from Veryan Medical Ltd.

\section{Funding}

This study was funded by Veryan Medical Ltd., Horsham, UK.

\section{ORCID}

Michael Lichtenberg

(D) https://orcid.org/0000-0003-2647-9876

\section{Correspondence address}

Michael Lichtenberg, MD

Vascular Center

Klinikum Hochsauerland

Stolte Ley 5

59759 Arnsberg

Germany

klichte@gmx.net 